Proceedings

\title{
Atoms, molecules, photons and phonons in primary science ${ }^{\dagger}$
}

\section{David Wood}

Publisher's Note: MDPI stays neutral with regard to jurisdictional claims in published maps and institutional affiliations.

\section{(c) (i)}

Copyright: (c) 2021 by the authors. Submitted for possible open access publication under the terms and conditions of the Creative Commons Attribution (CC BY) license (http://creativecommons.org/licenses /by/4.0/).

Abstract: Traditional science education wisdom suggests that abstract concepts are beyond the majority of primary-school aged students. This includes introducing atoms and molecules.

The Einstein-First curriculum introduces atoms and molecules into Year 3 via songs, role plays and simple atomic models made from plasticine (modelling clay) and balls with embedded magnets. They use these models and analogies to relate what they see at a macroscopic level to the miniscule structures of atoms and molecules, and the electrical forces that hold them together. At this early stage, we concentrate particularly on students becoming familiar with the language of modern science: atoms, molecules and photons.

The challenge is to introduce an atomic model that is faithful to the quantum and probabilistic nature of atoms and yet avoids both the misconceptions of planetary type orbitals which dominate almost all introductory chemistry. Instead, we show easily accessible images from the internet of the beautiful complexity of electron orbitals. We present atoms as a miniscule nucleus of protons and neutrons surrounded by an electron cloud in which the electrons are 'in there somewhere'.

This presentation will concisely outline our spiral learning approach in which students in Years 3 to 6 will revisit and develop concepts throughout four years of primary education. They will leave primary school with clear concepts of photons and phonons, changes of state (Year 3), physical properties of materials (Year 4), states of matter (Year 5) and simple reversible and irreversible changes to materials.

Keywords: modern science, primary school 